\title{
Conflit et territorialisation
}

\section{Conflicts and territories}

\author{
Marc Mormont* \\ SocioEconomie Environnement Développement \\ Faculté des Sciences \\ Université de Liège
}

\section{Résumé}

Un grand nombre des conflits observables dans l'espace rural se présentent aujourd'hui comme des conflits d'environnement. La mobilisation par les acteurs de ce répertoire est - elle simplement une rhétorique qui cache d'autres enjeux ou bien y a-t-il une spécificité, voire une nouveauté, dans cette mise en forme «environnementale » des tensions autour des usages de l'espace ? Cet article défend le second point de vue et propose une grille sociologique originale de lecture de ces conflits.

Les analyses classiques analysent les conflits d'espace comme conflits entre groupes sociaux, comme conflits d'appropriation de l'espace. Mais la réalité des conflits d'environnement porte sur d'autres enjeux qui ne peuvent apparaître qu'en analysant la dynamique de ces conflits. Dès lors cette contribution propose d'abord d'analyser ces conflits comme des conflits de cadrage c'est-à-dire comme portant sur la construction des objets mêmes des conflits. Ensuite l'analyse porte sur la dynamique des conflits, c'est-à-dire sur des processus où l'action collective tend à redéfinir ces cadrages en recomposant les normes, les connaissances et les identités à partir de quoi de nouveaux espaces sont redéfinis. Dès lors l'objet des conflits doit être redéfini : au-delà des objets concrets, des caractéristiques des environnements, ce sont des agencements et des territoires qui sont en jeu.

(C) 2006 Lavoisier, Paris. Tous droits réservés.

\section{Summary}

A lot of conflicts that are observed in rural areas can be qualified as environmental conflicts. The question is whether environmental arguments mobilised by social actors are to be considered as a

\footnotetext{
*Adresse email : mmormont@ulg.ac.be

(C) 2006 Lavoisier, Paris. Tous droits réservés.
} 
sort of rhetoric with some hidden interests or if there is a specificity or a novelty in these environmental discourses around nature, pollution or landscape and biodiversity. This paper defends the second assumption.

Current analysis consider conflicts around rural territories as conflicts between social groups that are competing to use space according their own social or economic priorities. But when we describe environmental conflicts, we can see other stakes that emerge from the social dynamics of conflict. Thus this contribution suggests to analyse these conflicts as framing conflicts that aim at redefining these objects. Then our analysis tries to describe the social dynamics, the specific processes of social alliances and oppositions that lead to draw up new framings. These processes imply a new norms, new knowledges or techniques, and transformed identities in order to rebuild territories. Beyond the concrete objects that are at stake, this paper propose a new grid to analyse territories by borrowing concepts to the deleuzian philosophy.

(C) 2006 Lavoisier, Paris. Tous droits réservés.

Mots clés : Conflits, territoires, espace rural, cadrage, débordement

Keywords: Conflicts, territories, rural area, framing, outflanking

\section{Introduction : conflits d'usage et conflits d'environnement}

Les conflits dans l'espace rural semblent se multiplier. Ces conflits sont à la fois des conflits entre usagers de l'espace rural, mais ce sont aussi des conflits entre usagers ou habitants et politiques publiques notamment à propos de décisions d'implantations d'équipements ou d'infrastructures ou encore à propos de programmes d'action publique. Ils portent non seulement sur des usages mais aussi sur des biens collectifs ; ils concernent des aspects du cadre de vie mais aussi des questions de distribution d'avantages et de risques (Aubin, 2004).

Ces conflits ne sont pas aussi récents qu'on pourrait le croire : ils sont l'objet d'analyses sociologiques depuis les années soixante - dix, soit à l'époque où les espaces ruraux cessent d'être des espaces agraires d'émigration. Dès cette époque en effet le développement du tourisme, des résidences secondaires et les débuts de la rurbanisation opèrent plusieurs changements importants : d'une part de nouvelles populations, d'origine urbaine, temporaires ou permanentes, fréquentent des espaces ruraux ; d'autre part ces espaces n'ont pas pour ces nouveaux occupants un statut de ressource productive (agricole), mais une fonction de cadre de vie; enfin de nouvelles politiques publiques ou initiatives privées confèrent aux espaces ruraux de nouvelles fonctions de préservation de ressource, qu'il s'agisse de nature puis de biodiversité ou bien de ressources naturelles comme l'eau.

Il est un autre changement important : c'est que les conflits qui se développent renvoient, de manières souvent différentes, au discours environnemental. Nombre des politiques publiques évoquées ci-dessus se revendiquent en effet de la préservation de l'environnement, mais les opposants à des implantations industrielles ou au développement de l'agriculture intensive se revendiquent également défenseurs de l'environnement. S'agit-il simplement d'une rhétorique qui permet de donner à des préoccupations particulières, intérêts particuliers ou sectoriels, une dimension d'intérêt collectif ou s'agit-il 
d'un nouveau champ de conflictualité qui est différent des conflictualités classiques de la société industrielle ? La conflictualité classique dans l'espace rural opposait à la fois des groupes sociaux pour l'appropriation de l'espace et des sociétés locales à l'Etat pour le contrôle des ressources productives. La conflictualité environnementale est-elle d'une autre nature ? Nous défendrons l'idée que c'est bien le cas et que cela impose de développer une autre grille de lecture des conflits d'espace, qui sera l'objet de cet article.

\section{L'analyse sociologique des conflits d'espace et d'environnement}

Dès les années soixante-dix des conflits d'usage émergent dans les zones rurales, conflits qui ne concernent plus seulement des questions agricoles ou forestières mais bien des questions d'affectation des espaces. Cette période est appelée, dans le langage sociologique de l'époque, celle de l'urbanisation des campagnes. Cette urbanisation est caractérisée comme l'équivalent, dans le domaine des modes de vie ou de la culture, de la modernisation agricole qui introduit, elle aussi, de nouvelles manières de caractériser à la fois l'espace (la pierre de touche est le remembrement agricole), le métier (de mode de vie, l'agriculture devient une profession) et donc les identités. La séparation qui s'opère progressivement entre la ferme et le logement en est une traduction.

Cette dynamique d'urbanisation se traduit aussi par l'entrée dans les zones rurales de nouvelles populations, de nouveaux usages de l'espace et de nouveaux acteurs. Le développement du tourisme en est l'illustration la plus frappante à l'époque : à travers le tourisme ce sont des populations urbaines et des populations rurales qui se confrontent et parfois s'affrontent. L'espace rural comme espace récréatif entre en tension avec l'espace productif des agriculteurs. Des entrepreneurs touristiques entrent en scène et en concurrence avec les propriétaires des terres agricoles. Les stations touristiques, notamment les stations de ski, introduisent à la campagne à la fois une autre conception de l'espace mais aussi la division du temps en temps de loisir et temps de travail.

Alors que les économistes et certains sociologues situent l'origine de ces conflits dans l'affrontement entre des fonctions différentes (fonction productive, fonction récréative, etc.), nombre de sociologues y voient plutôt la confrontation entre des ensembles sociaux qu'on peut assez facilement caractériser comme ruraux d'un côté et urbains de l'autre. On est là dans une perspective plutôt culturaliste qui décrit un processus de modernisation qui n'est pas seulement technique et économique mais aussi socioculturelle puisqu'elle fait entrer les sociétés agraires (certains disent paysannes) dans la modernité. Les conflits peuvent alors s'interpréter en termes de résistance à ce processus.

Une autre grande ligne d'interprétation va se développer, notamment en France, pour analyser ce même processus et ces conflits en termes de conflit social. Dans cette perspective, notamment inscrite dans l'école de Bourdieu, ces conflits opposent en fait des groupes sociaux, voire des classes sociales. Les enjeux de ces conflits sont à la fois des enjeux culturels et des enjeux d'appropriation. Les enjeux culturels concernent ici moins l'accès à la culture savante que des tensions entre des habitus : les cultures paysannes sont dévalorisées, elles perdent droit à l'expression. Les systèmes de valeurs et leur mode d'expression dans des usages et des pratiques quotidiennes sont disqualifiés et avec eux ceux pour qui elles avaient du sens. Quant aux enjeux d'appropriation, ils peuvent mieux se définir comme les droits d'accès à des ressources. C'est ici que l'environnement - même 
si le terme est moins utilisé à l'époque qu'aujourd'hui - intervient une première fois car il constitue une manière de légitimer des modes d'usage de l'espace.

Dans cette perspective, et c'est ce qui nous importe ici, on tend nécessairement à mettre en évidence des incompatibilités entre des usages de l'espace qui sont propres à des groupes sociaux. Ces tensions ne concernent pas seulement des tensions entre ruraux et citadins, car les conflits peuvent aussi opposer des classes urbaines pour l'appropriation de l'espace rural. Celui-ci est devenu un enjeu dans les rapports entre des couches sociales tant urbaines que rurales.

Un exemple typique de ce type d'analyse peut être résumé de manière stylisée (Mormont, 1980). Dans une vallée ardennaise pittoresque, le tourisme s'est développé dans les années soixante de manière relativement anarchique sous forme d'une série de campings fréquentés par des couches populaires. Sur les pentes des collines qui encadrent cette vallée se sont aussi multipliées les résidences secondaires fréquentées par une population plus aisée et plus cultivée qui privilégie le calme, la nature. Ces derniers ont une vision esthétisante et naturaliste de la campagne qui fait contraste avec les demandes plus ludiques de la clientèle populaire des campings. Les premiers sont amateurs de paysage, les seconds semblent préférer le jeu, la joyeuse convivialité un peu bruyante. Or les premiers vont s'appuyer sur cette vision esthétisante de la campagne, sur des arguments écologiques même pour défendre l'idée d'une protection de cette vallée. Les premières législations d'aménagement leur donnent des outils pour exiger le déménagement des campings dont la légalité n'est pas certaine. Les campings enlaidissent le paysage, polluent la rivière et le site, le bruit trouble la quiétude de la nature. Les ruraux vont se diviser dans ce conflit notamment entre ceux qui voient dans ce tourisme de masse une possibilité de développement économique et ceux qui au contraire y voient une menace pour leurs activités agricoles ou parfois pour la bonne moralité. De curieuses alliances se nouent donc. Mais ce conflit est bien analysable en termes d'oppositions entre des cultures de classe. Ce sont des représentations différentes de l'espace rural qui s'opposent, représentations associées à des ethos de classe : l'enjeu est donc fondamentalement un enjeu culturel. Si une représentation de l'espace (comme paysage, comme nature à préserver) s'impose, elle favorise certaines catégories sociales au détriment d'autres qui en seront exclues. Dans cette perspective la rhétorique environnementale apparaît comme un discours qui vient légitimer un mode d'usage de l'espace.

Dès lors, le conflit est donc aussi conflit symbolique et politique. Ce qui va jouer un rôle décisif dans ce conflit c'est la capacité relative des acteurs d'imposer des catégories de représentation de l'espace comme les catégories légitimes : la définition, à travers des législations revendiquées par des acteurs collectifs comme les associations de défense de l'environnement ou du paysage, de l'espace rural comme espace protégé, définition qui mêle des catégories naturalistes et esthétiques, est évidemment une définition cruciale pour l'issue du conflit. Le conflit local - celui qui concerne cette vallée - est à la fois la manifestation d'un conflit d'intérêts (culturels et symboliques), mais aussi un processus dans lequel s'élabore et se mettent en forme des argumentations, des coalitions sociales, qui, médiatisées par des processus politiques, vont conduire à des normes légales, des procédures d'évaluation, qui vont finalement sanctionner, à l'échelle nationale, le rapport de forces entre les parties en présence. Par suite les politiques publiques, par exemple celle de protection du paysage, inscrivent alors dans l'ordre institutionnel légitime cette inégalité des groupes sociaux. 
De telles analyses sont relativement proches de celles qui ont trait à des formes de conflictualité dans l'espace urbain. Ainsi Chamboredon (1970) pouvait analyser les conflits de voisinage, par exemple autour du bruit, comme des conflits qui renvoyaient finalement à des modes de vie différents et à la concurrence symbolique entre ces cultures quotidiennes. De même Boltanski (1975) analysait les tensions entre conducteurs d'automobile comme expression d'un conflit de classe médiatisé par les conditions spécifiques et inégales dans lesquelles des agents appartenant à des catégories sociales inégalement dotées accèdent à un moyen de transport.

Cette perspective d'analyse sociologique des conflits d'espace se focalise donc sur un type d'enjeu spécifique c'est-à-dire l'espace en tant que ressource. Il ne s'agit pas ici tellement d'une ressource économique, mais plutôt de l'espace comme ressource accessible différentiellement pour l'expression d'une culture ou, dans un langage autre, d'un style de vie. Les conflits quotidiens, ceux de voisinage, comme les conflits relevant de l'action collective, sont justiciables au même titre de telles analyses. Ce sont finalement des conflits de légitimité : l'espace étant une ressource relativement rare, il y a concurrence pour son appropriation entre des modes d'usage qui sont peu ou pas compatibles. Il s'agit alors de faire prévaloir des usages légitimes. Et les politiques publiques, spécialement celles qui organisent l'aménagement du territoire, sont évidemment cruciales pour consacrer la légitimité des usages.

Quant à l'environnement il est clair qu'il ne s'agit pas ici d'un enjeu, c'est plutôt un instrument, une ressource dans le processus de légitimation : qu'il s'agisse de paysage, c'est dans la culture savante ou semi-savante qu'on trouve des ressources pour protéger une patrimoine commun, qu'il s'agisse de nature, c'est dans les sciences naturelles qu'on va trouver appui pour demander la protection d'un écosystème, mais ces ressources ne sont pas des enjeux ce sont des instruments mobilisés au service d'intérêts autres, d'intérêts proprement sociaux.

Ce type d'analyse sociologique n'est contestable ni dans son principe, ni dans ses résultats. Mais ces analyses reposent sur deux considérations implicites qui, me semblet-il, les rendent insuffisantes pour comprendre la conflictualité environnementale dans l'espace rural. D'une part elles supposent qu'on puisse identifier dans l'analyse des correspondances claires entre des intérêts sociaux (économique ou culturels) et des usages de l'espace (Mormont, 1990). D'autre part elles supposent qu'on puisse réduire les enjeux environnementaux à un statut instrumental, donc à un rôle de ressource argumentative et non au statut d'enjeu. Cette double réduction ne me paraît plus suffisante aujourd'hui.

\section{Des conflits de cadrage}

Les analyses sociologiques évoquées ci-dessus supposent en effet que le cadre d'interprétation de l'analyste (par exemples celui d'un conflit rural / urbain, ou celui d'un conflit de cultures de classe) puisse être mis en correspondance avec les pratiques observables des acteurs. Si l'observateur peut mettre en correspondance, à travers une série d'indicateurs, des manières d'utiliser l'espace (usage du temps, sociabilité, rapport à la nature, etc.) avec des propriétés pertinentes d'appartenance sociale des acteurs, il inscrit alors le conflit dans un cadre cohérent. Cette démarche suppose cependant que le cadrage du sociologue puisse être mis en rapport avec celui des acteurs : c'est le cas dans l'exemple évoqué ci dessus quand les campeurs expulsés au nom de la protection du paysage mani- 
festent en proclamant «nous sommes aussi beaux que des vaches » : le sociologue peut alors y voir une protestation populaire contre l'esthétisme des protecteurs du paysage. Dans ce cas la correspondance entre le cadrage du sociologue (conflit de légitimité pour l'appropriation de l'espace) peut ainsi être établie avec le cadrage spontané des acteurs même si celui-ci est bien plus simple, incomplet que celui de l'analyste. Dans cette perspective on peut même rendre compte des faiblesses ou des erreurs du cadrage opérées par les acteurs, si on peut en rendre compte à l'intérieur du cadre d'analyse sociologique : ce serait par exemple le cas si les acteurs populaires, qui fréquentent l'espace rural de manière beaucoup plus temporaire et plus occasionnelle, disposaient de beaucoup moins de moyens sociaux et culturels pour peser sur la régulation des usages de l'espace alors que les résidents secondaires peuvent plus facilement tisser un réseau de relations locales capables de peser sur les décisions et qu'ils disposent des ressources langagières et argumentaires pour mobiliser la rhétorique de l'environnement. Dès lors l'environnement est toujours réinterprété, par le sociologue, et ramené ou réduit à sa signification sociale.

En d'autres termes l'analyse sociologique inscrit le cadrage de l'action des acteurs dans un cadre d'analyse qui lui est propre mais en les mettant en correspondance. Il y a là une double exigence : celle de la capacité du cadrage sociologique à rendre compte du conflit, et celle de la mise en relation de ce cadre avec celui qu'opèrent les acteurs euxmêmes. La première exigence est celle de pouvoir définir les enjeux du conflit pour les différents acteurs, la distribution des atouts dans le jeu, tandis que la seconde implique de pouvoir expliquer comment les acteurs interprètent le conflit et les situations dans lesquelles ils sont engagés.

Qu'en est-il des conflits d'environnement?

Nous faisons l'hypothèse que les conflits d'environnement ne peuvent plus relever seulement d'une telle analyse parce que ce sont précisément des conflits qui portent sur le cadrage des situations et des objets de conflits, c'est-à-dire sur la définition même de ce qui est en jeu, sur la définition des paramètres pertinents, qu'il s'agisse de connaissances, de normes et même des identités des acteurs en présence.

Nombre de conflits opposent des riverains, des habitants, à des promoteurs d'une nouvelle infrastructure, publique ou privée, au nom de leur quiétude ou de leur sécurité ou plus simplement de leur cadre de vie. Les qualifier de Nimby (not in my backyard) c'est précisément une opération de cadrage : celle-ci consiste à les considérer comme défendant des intérêts particuliers, locaux et individuels, et à les mettre en opposition avec les intérêts collectifs, généraux, qui sous-tendent par exemple l'installation nécessaire d'une usine de traitement des déchets pour un vaste territoire. Un tel cadrage du conflit - souvent opéré d'ailleurs par certains acteurs eux-mêmes - a évidemment des effets sur le conflit lui-même puisqu'il tend à disqualifier certaines actions (égoïstes).

S'agissant par exemple des conflits sur les usages des forêts, nous pouvons observer des situations plus complexes. La gestion des forêts communales en particulier a fait l'objet, il y a plusieurs décennies, de dispositions qui fondent leur gestion par l'administration sur un double souci : préserver et valoriser une ressource productive dont les revenus sont attribués aux collectivités locales, laisser à celles-ci un pouvoir de négociation des autres usages (chasse, loisir) avec les autres usagers dans le respect des contraintes de «productivité ». Est ainsi érigé un cadre institutionnel qui désigne des identités (usagers légitimes), formule des droits d'usage et d'accès pour 
les uns et les autres, et s'appuie sur un corps de connaissances relativement stabilisées. Dans ce cadre institué, on peut évidemment définir les intérêts en jeu et y rapporter les conflits observables par exemple entre promeneurs et chasseurs. On a bien affaire à des conflits d'appropriation qui sont cadrés par le système institutionnel, lequel donne lieu évidemment à une variété de configurations locales selon les états de la forêt, les morphologies sociales, les traditions enracinées, etc.

L'irruption d'un acteur nouveau ( les associations naturalistes) ou d'une nouvelle politiques (Natura 2000 ou la loi sur l'eau) va se traduire par de nouveaux conflits, qu'on peut bien sûr interpréter comme des conflits qui résultent simplement de l'émergence de nouveaux acteurs ou de nouveaux usages ou encore de nouvelles fonctions de la forêt. L'analyse peut alors se déployer classiquement par l'identification des enjeux, l'analyse des alliances et des compromis éventuels, De ces conflits peuvent résulter des hégémonies nouvelles ou des exclusions. On pourra éventuellement rapporter ces jeux à des conflits entre vocations des espaces ou entre groupes sociaux qu'il s'agisse de catégories sociales ou de groupes professionnels dont les intérêts et les préférences sont liés à des affectations ou des usages définis de l'espace forestier.

Néanmoins on s'aperçoit que ces conflits ne se résument à des conflits d'usage que si on peut définir les intérêts en présence de manière à définir une configuration relativement stabilisée des acteurs et des enjeux. Cela ne peut se faire que par un cadrage du conflit qui va par exemple assigner aux naturalistes un intérêt sectoriel ou professionnel opposable aux intérêts des forestiers ou à ceux des collectivités locales. Or c'est précisément ce qui est souvent en jeu dans ces conflits : la diversité biologique de la forêt est-elle un intérêt spécifique parmi d'autres, assignable à certains acteurs qui portent cet enjeu, avec lequel il faut éventuellement composer (par exemple en opérant un zonage de la forêt) ou est-elle une autre manière de voir l'ensemble des usages de la forêt en ce y compris la production de bois, la chasse et le loisir. Tout cela dépend en fait du cadrage auquel on se réfère.

On pourrait donc par exemple considérer un tel conflit comme un conflit local et ponctuel, qui oppose des usages différents, et des groupes d'usagers différents (forestiers, chasseurs, naturalistes, randonneurs, élus locaux). Si ce changement laisse chasseurs, forestiers et collectivités indemnes, c'est-à-dire qu'il ne change ni leurs pratiques, ni leurs préférences, ni leurs identités, le changement est à la marge. Les acteurs s'adaptent et on peut interpréter ces changements simplement à partir des logiques instrumentales où les acteurs mobiliseraient des ressources juridiques ou cognitives pour promouvoir leurs intérêts. Mais la réalité des conflits observables c'est que certains acteurs ne veulent pas s'arrêter là : pour certains la protection de telle espèce est un enjeu national ou international et ils s'appuient pour cela sur des législations européennes de protection de la nature, ils veulent aussi redéfinir la gestion forestière comme un défi à long terme en la liant par exemple à la problématique du changement climatique et ils s'appuieront pour cela sur des connaissances nouvelles qui ne sont pas celles qui sont familières aux forestiers... Dès lors ce conflit pourrait aussi être un cas particulier du processus général qui conduit d'une forêt économique à une forêt multifonctionnelle et partagée entre des fonctions différentes. Car ce qu'ils font ainsi ce n'est pas seulement mobiliser des ressources mais construire autrement la situation et l'objet du conflit. Et c'est aussi redéfinir les intérêts voire les identités des autres acteurs.

En effet une forêt multifonctionnelle qu'est-ce d'autre qu'un nouveau cadre pour penser la gestion forestière ? Cadre dans lequel certains usagers (loisir, nature, biodiversité) 
prennent une place légitime, acquièrent le statut d'interlocuteurs, se voient reconnaître des droits... Si les conflits localisés ne sont pas une pathologie de l'ordre social, mais une des modalités de construction de l'action collective (ou de la coordination) c'est bien parce qu'ils sont producteurs de cadrage de cette action collective.

Le concept de cadrage trouve son origine chez Goffman (1974) : dans sa perspective d'analyse des interactions ordinaires, il cherchait à décrire la manière dont un acteur définit la situation dans laquelle il s'engage, relie les éléments singuliers à d'autres qui dépassent cette situation et se construit ainsi un espace de communication avec autrui. Ce concept a été repris par la sociologie politique tant du côté de la sociologie de l'action collective (Snow et al., 1986) que de la sociologie des politiques publiques ou encore dans l'analyse de la communication politique (par exemple Neveu, 1999). Beaucoup de ces analyses font des cadrages des opérations stratégiques par lesquelles certains acteurs imposent des orientations à la pensée et à l'action politiques. Même si certains accordent une importance aux conflits de cadrage (Benford et Hunt, 2001), on reste dans une perspective où le cadre est plutôt un instrument de l'action politique et où on identifie cadrage et acteur.

A partir de la notion de traduction, Callon (1999) va redonner une impulsion à ce concept en en faisant à la fois un résultat et un opérateur de traduction entre des mondes différents, par exemple ceux de l'environnement et du marché : c'est dans cette perspective plus dynamique, où cadrages et acteurs évoluent ensemble, que s'inscrit cette analyse.

Le cadrage d'un problème, c'est l'ensemble des opérations par lesquelles se définissent les paramètres de sa définition, le temps et l'espace pertinents, mais aussi la place des acteurs, les intérêts en jeu. Tout cadrage est prise en compte, il définit ce qui compte dans la situation mais le cadrage a aussi un effet d'effacement de ce qui n'est pas pris en considération. C'est ce qui permet de le relier au concept d'externalité (Callon, 1998). Ce qui se produit, dans nombre de ces conflits, ce n'est pas seulement un conflit entre des usages et des intérêts liés à ces usages, donc des conflits d'externalités, ce sont des conflits de cadrage des conflits d'usage, de définition de ce qui compte et de comment le prendre en compte. Par conflit de cadrage on entend le conflit en tant qu'il porte sur la définition même des paramètres qui servent à le définir, à situer ses enjeux, à caractériser les intérêts et les groupes pertinents, donc à définir les acteurs.

Tout cadrage s'opère par la mobilisation simultanée de connaissances, de normes et d'identités réelles ou potentielles des acteurs. Ainsi une certaine manière de comprendre l'écologie forestière peut permettre d'assigner aux chasseurs (ou à certains d'entre eux de revendiquer) une fonction de gestion écologique de la faune s'ils acceptent certaines normes de gestion. De même si on mobilise à la fois des ressources monétaires, des connaissances techniques et un enjeu écologique comme l'eau souterraine on peut très bien redéfinir l'agriculteur comme gestionnaire de la ressource. Le cadrage est donc solidaire d'une triple composition des savoirs, des normes et des identités.

Cette notion de cadrage permet de comprendre les ambivalences (Claeys-Mekdade, 2003) des acteurs dans l'action collective, par exemple le fait que les élus locaux peuvent, en fonction du cadrage de la situation, modifier complètement leurs objectifs et leurs stratégies. Les tensions qui émergent aux points de friction entre des usages peu compatibles de l'espace peuvent en effet faire l'objet de plusieurs interprétations selon le cadre dans lequel les échanges entre les acteurs vont situer ces enjeux et ces identités. Cette ambivalence est un des ressorts de l'action collective qui se déploie dans ces conflits. 


\section{La dynamique des conflits}

Dans certaines traditions sociologiques notamment inspirées de Simmel (1908a et b), le conflit n'est pas seulement un révélateur de tensions sociales sous-jacentes. En observant le déroulement du conflit, l'analyse peut dégager des effets propres de transformation mutuelle des acteurs du fait de leurs interactions : des règles nouvelles apparaissent du fait même de ces interactions. Les conflits sont aussi des formes de socialisation. Mais cette création de société est souvent analysée au niveau même des interactions, des relations d'échange et de négociation entre les acteurs dans l'espace même du conflit. C'est aussi ce qui donne lieu à toutes les théories mi descriptives, mi normatives de la concertation ou de la négociation. Sans négliger cette ligne d'analyse, nous faisons l'hypothèse que si les conflits sont créateurs, c'est aussi par une autre voie, plus complexe : celle du recadrage et du réagencement.

Par dynamique des conflits nous désignons le processus qui va du déclenchement de l'action jusqu'à l'éventuelle résolution. Nous ne nous intéressons pas ici aux seules modalités (négociation, arbitrage) de résolution locale des conflits mais plutôt à ce qu'on pourrait appeler leur productivité, c'est-à-dire aux effets des conflits. Nous faisons l'hypothèse que, au moins dans certains cas jugés les plus intéressants ou les plus productifs, les conflits ont des effets de cadrage ou de recadrage, donc de reformulation des problèmes; que ces opérations de recadrage sont rendues possibles par l'action collective et qu'enfin ces recadrages peuvent déboucher sur des reconfigurations territoriales.

L'analyse du conflit suppose une conception de l'acteur social. L'action collective est le plus souvent analysée par les sociologues en termes d'action stratégique. Cette définition de l'acteur suppose un acteur capable de se donner des objectifs, de développer des stratégies, d'évaluer son action et ses résultats, de passer des accords ou des compromis. C'est une conception forte de l'acteur qui exige de lui des compétences étendues. Pour nous, stratégie il y a, mais il ne s'agit pas nécessairement de stratégie au sens d'un choix conscient et raisonné des moyens par rapport à des fins. L'acteur est stratégique dans un sens à la fois plus faible et plus fort : nous supposons plus faiblement que les acteurs ont un sens stratégique plus qu'une stratégie, c'est-à-dire une compétence à anticiper et à évaluer, mais que cette compétence ne peut s'exercer que dans un cadrage de l'action et des interactions c'est-à-dire en s'appuyant sur des ressources extérieures. Mais cette compétence stratégique est aussi une capacité forte en ce sens qu'elle peut être une capacité à sortir d'un cadrage, à en changer, à en mobiliser plusieurs au cours de l'action. Une telle définition permet de penser un acteur mobile (multi-stratège), ambivalent (jouant plusieurs jeux) voire rusé (affichant une stratégie tout en en poursuivant une autre). Mais comme cet acteur doit s'appuyer sur des ressources (cognitives, normatives voire identitaires), son action consiste aussi à chercher et construire ces ressources dans l'action ellemême. Ce faisant il se construit lui-même, redéfinit ses objectifs, redéfinit son identité.

Dans les conflits observés ${ }^{1}$, la première étape est souvent celle d'une protestation à l'égard d'une action d'autrui, d'un projet porté par un acteur. L'opposant se pose alors en victime. Mais l'individu seul ne peut guère. Il lui faut s'associer, trouver d'autres sembla-

\footnotetext{
${ }^{1}$ Nous ne pouvons donner ici que des descriptions très stylisées de conflits que nous avons pu étudier dans le cours de différentes recherches.
} 
bles. Cette association première peut s'appuyer parfois sur des cadres institués (comme le syndicat professionnel, l'association de chasse) mais souvent elle passe par un premier cadrage qui se base sur des normes générales et des imputations de risque : le droit à un environnement sain et le risque de pollution, par exemple. L'action se réfère alors à un « nous » de victimes ou de droits bafoués. Ceci donne ainsi souvent au point de départ de l'action le caractère d'une action sinon égoïste du moins de défense de droits ou d'acquis. Le projet d'implantation d'une station d'épuration à quelques dizaines de mètres d'un lotissement résidentiel suscite la crainte des habitants : défense d'une aménité privilégiée ou revendication environnementale légitime? Le comité de riverains qui se constitue joue stratégiquement de tous les arguments. Mais seul il est faible face aux arguments techniques - une localisation idéale à proximité de la rivière - et politiques - l'intérêt collectif, l'environnement mieux protégé. On peut même l'accuser de vouloir déplacer la pollution vers un autre quartier de la commune. Il se cherche donc des alliés.

L'existence à proximité d'une réserve naturelle dûment protégée offre une opportunité. L'association naturaliste qui gère cette réserve s'en inquiète. Elle fait appel à la fédération régionale des associations d'environnement qui apporte à l'action ses compétences autant que son poids politique. Ce renfort n'est pas seulement addition d'une force à une autre : il modifie aussi le cours de l'action et le registre des argumentations. Les environnementalistes introduisent en effet des arguments nouveaux : la station d'épuration est localisée dans une zone humide ; leur expertise en aménagement les amène à se pencher sur le plan d'occupation des sols et à chercher une localisation plus adéquate selon de nouveaux critères. Ce faisant les arguments changent : l'espace n'est plus seulement le quartier, le groupe n'est plus seulement celui des riverains, il devient celui des associations locales. Dès lors il n'est plus possible, si on veut associer d'autres comités de quartier, de simplement renvoyer le projet vers un autre quartier. L'action se déplace alors vers une critique serrée des critères de localisation : si une localisation en fond de vallée est raisonnable pour des raisons hydrauliques, on s'aperçoit qu'un autre site est possible, celui d'une ancienne usine de batteries, abandonnée depuis deux décennies. Ce site permettrait même de réaliser un réseau d'égouttage moins cher sans abîmer la vallée. Par contre ce nouveau choix pose d'autres problèmes : cela supposerait de dépolluer le site contaminé par le plomb mais le propriétaire s'est évanoui dans la nature. Dès lors l'autorité locale, jusque là solidaire de l'auteur de projet, voit un intérêt nouveau à l'affaire ; ce serait l'occasion de réhabiliter ce site industriel et les riverains de ce nouveau site y sont favorables. Se crée alors une nouvelle coalition qui va devoir surmonter deux obstacles : celui de la méconnaissance des techniques de dépollution des sols ; celui des droits de propriété du site industriel. Pour y parvenir la nouvelle coalition va s'adjoindre de nouveaux partenaires au niveau régional. Des experts de dépollution des sols valident l'option. Les critères de localisation de la station d'épuration sont revus et le propriétaire du site est exproprié. Mais de cette nouvelle coalition sort aussi un nouveau projet sur le territoire communal combinant réaménagement d'un site industriel, implantation d'une station d'épuration et élargissement de la protection des zones humides. Cette description stylisée n'est pas seulement celle d'une négociation locale entre des intérêts prédéfinis.

Le conflit était au départ micro-local, une simple question d'accord / désaccord sur la localisation d'un équipement. Au terme du processus, on a mobilisé d'autres acteurs, 
d'autres connaissances, formé d'autres coalitions, on a modifié des normes techniques et revu le plan d'occupation des sols. Les acteurs ne sont plus les mêmes et les relations de ceux-ci au territoire, sinon les identités, ont changé.

Cela n'a pu se faire que par des opérations de cadrage et de recadrage de l'action et du conflit. Le recadrage s'opère toujours par conjugaison de forces et, comme en grammaire, qui dit conjugaison dit changement de pronom (qui parle ?), de temps, de modes, de personnes. Et ces processus déplacent ainsi des conflits d'espace (au sens de conflits de coexistence spatiale entre des activités et des acteurs) vers des conflits plus complexes, parfois plus généraux, mais aussi reviennent sur des sites et des actions ponctuelles. Les conflits sont donc traités comme des événements potentiellement transformateurs (pour le concept de transfromative event, voir Aminzade et al., 2001).

Il importe par conséquent d'analyser les conflits en suivant la circulation des questions, des arguments et les reconfigurations auxquelles cette circulation donne lieu. «Circulez, tout est à voir » pourrait être le titre de cette contribution, tant ces déplacements de l'action et les recadrages qui s'ensuivent nous donnent des indications sur les configurations sous-jacentes aux choix d'aménagement ou de localisation. Quatre composantes s'enchaînent en effet dans le déroulement de cette action, depuis la protestation de quelques riverains jusqu'à la redéfinition de l'aménagement local.

La première composante est un mouvement, un enchaînement de déplacements. Il consiste à passer d'un problème micro-local, la crainte des riverains de se voir pollués par une station laide et malodorante, à des questions supra-locales, celle des critères d'optimisation de la localisation des stations ou celle des sites industriels désaffectés d'une nuisance à des environnements, au paysage, aux sites industriels.... Ces déplacements ne peuvent se faire que via des associations, qu'en créant des coalitions nouvelles, qu'en intéressant des naturalistes, d'autres comités de quartiers, d'autres experts aussi. Ces déplacements par associations successives transforment évidemment l'acteur collectif, sa dimension sociale et spatiale. Mais aussi ce sont ses arguments et les identités auxquelles il est fait appel qui changent. Chacune des étapes comporte pour l'acteur originel des opportunités d'accroître son poids politique, mais aussi des risques : à mesure qu'il s'associe à d'autres, il doit accepter d'inscrire son action dans une autre action, et le risque est de voir par exemple les naturalistes concéder que cette localisation n'est pas négative pour eux. Chaque association transforme aussi les arguments utilisables : s'associer à d'autres comités de quartiers interdit d'exiger que d'autres subissent les mêmes nuisances dont on ne veut pas. Il faut trouver des arguments plus nombreux mais aussi plus généraux. Cette recherche de généralité se fait en remontant dans les circuits institutionnels et cognitifs des décisions. On passe du quartier à la commune, de la commune à la Région ${ }^{2}$, etc. On passe aussi de données locales à des méthodes de calcul.

L'action, par association d'alliés, ajoute des prises en compte. On s'aperçoit par exemple que la localisation de la station oublie la question du paysage. En parlant aux associations de la commune voisine, on s'aperçoit aussi que la manière de calculer les coûts de la station est

2 L'expérience exemplaire de l'action de Eaux et Rivières de Bretagne est ici très éclairante : protestant contre la pollution des eaux de surface, cette association a fini par remonter, via la voie judiciaire, aux options de l'Etat français et de la politique agricole commune qui ont été condamnées pour les encouragements qu'elle procurent au développement d'une agriculture intensive (Mallard et Remy, 1999). 
faire indépendamment des communes voisines : or la nouvelle localisation permettrait de faire une seule station pour deux agglomérations et ainsi de réaliser une économie substantielle ; nouvelle prise en compte, à une autre échelle de calcul. C'est la dynamique même de l'action collective qui provoque ces déplacements. Car la recherche d'alliés, la constitution progressive de coalitions entraîne l'action au-delà du local. Elle modifie à la fois les paramètres, les échelles, élargit à de nouveaux objets via de nouveaux porte-parole.

Une deuxième composante du processus consiste en effet à se procurer, à travers ces déplacements, des ressources nouvelles. L'argument de départ est simple, relatif simplement à la gêne subie et au droit à un cadre de vie protégé. Cet argument est fort car il est facilement partagé par tous dans un registre de sens commun où chacun a droit à cette quiétude. Mais cette force est aussi une faiblesse, car cette valeur partagée peut aussi opposer tous ceux qui la partagent : quel habitant voudrait de cet équipement au fond de son jardin? Les déplacements par associations ne permettent donc de renforcer l'argument que si d'autres connaissances et d'autres normes peuvent être mises en jeu. Ce sont des ressources nouvelles que procurent les associés. Des ressources cognitives d'abord : le calcul d'optimisation de la localisation peut être questionné pour montrer qu'en modifiant certains paramètres, on aboutit à d'autres choix. Des ressources normatives aussi : si une priorité est donnée par la région à la réaffectation des sites industriels désaffectés, alors le calcul ne se fait plus seulement au niveau de la station mais en combinant les deux projets dans un projet commun où l'investissement est autrement distribué. Des ressources identitaires enfin car il ne s'agit plus d'un quartier mais de la commune, voire de deux. La mobilisation de ces ressources, parce qu'elle introduit de nouveaux paramètres, est de nature à faire changer certains acteurs de stratégie : les autorités locales voient de nouvelles opportunités et changent de camp, toujours au nom de leur mandat électif.

Dès lors que d'autres ressources permettent d'autres manières de calculer, un nouveau cadrage s'impose peu à peu. D'un conflit classique entre riverains et projet «nuisant », on passe à un débat entre une commune et des pouvoirs supérieurs autour de la manière dont on peut aménager le territoire communal. Le conflit est recadré. L'action collective procède donc par un double mouvement de déconstruction du cadrage et de recadrage. Construire un nouveau cadre comporte une double opération. Il s'agit pour l'acteur en interaction avec d'autres de faire reconnaître d'autres paramètres d'évaluation, bref de faire admettre d'autres prises en compte. Et si ces prises en compte, par exemple de la nature pour les naturalistes, des sites pollués pour la commune ou la Région, peuvent valoir, c'est bien parce qu'elles procurent des opportunités stratégiques pour ces acteurs. L'association prend ici un caractère relativement calculé : le sens stratégique de l'acteur, c'est de saisir la convergence que ce nouveau cadre peut créer entre les opportunités stratégiques qu'il offre aux différents partenaires. Il s'agit ensuite de trouver un équilibre entre les différentes prises en compte, de pondérer les arguments, de créer des équivalences acceptables de telle manière que des concessions réciproques soient possibles.

Dans cette phase de la dynamique il peut être très difficile pour certains acteurs qui se sont constitués dans le cours de l'action d'accepter cet arbitrage et cette négociation. Le comité local de riverains qui s'est constitué contre le projet, souvent de manière radicale, peut éprouver de grandes difficultés à accepter une solution négociée dans laquelle il serait amené à reconnaître que le risque est finalement moins grand que ce qu'il avait affirmé au départ de l'action. Cet acteur devrait ainsi renoncer à des arguments qui étaient 
constitutifs de son identité de départ centrée sur un refus inconditionnel du projet. C'est ici que va jouer l'ambivalence évoquée ci-dessus. Car c'est seulement si cet acteur - à travers les liens noués au long des associations qui ont accru son poids et son envergure sociale - a pu intégrer divers autres arguments, qu'il pourra se mettre en mesure d'accepter une négociation. C'est qu'il s'agit souvent pour ces acteurs d'être plus que des acteurs locaux, d'être capables d'être à la fois des défenseurs d'un bien collectif local, mais aussi des défenseurs d'un bien collectif supérieur. Cela ne peut se faire que si l'issue du conflit crée pour eux de nouvelles opportunités.

La quatrième composante est en effet celle de la résolution du conflit, c'est aussi celle de la reconfiguration du local. Un autre exemple 3 peut être évoqué à ce propos. C'est celui d'une commune où l'industrie traditionnelle de la pierre est en déclin. Les autorités locales cherchent une reconversion économique qui maintienne cette tradition de la pierre et cherchent donc une valorisation touristique de ces grottes et carrières. Parallèlement s'est mis en place un forum local pour la biodiversité. Les naturalistes (amateurs et experts) procèdent à un inventaire des ressources et mettent à jour la présence de chauve-souris dans une cavité souterraine également convoitée par la commune qui veut la céder à une entreprise de loisirs sportifs. La situation est conflictuelle et une expertise indépendante est demandée. L'expert international convoqué révèle qu'il s'agit d'une espèce de chauve-souris si rare qu'elle donne une dimension internationale à cette grotte. La liste européenne des espèces protégées peut être convoquée. Changement d'objet, changement d'échelle. Ces connaissances inédites, l'appel possible à des normes supérieures induisent un débat politique et juridique entre autorités locales et régionales, entre administration du développement et de l'environnement, quant au statut à accorder à la grotte. La situation serait bloquée si le forum local ne permettait de nouer des contacts entre entrepreneurs locaux du tourisme, associations et autorités locales. Naturalistes et entrepreneurs de loisir se mettent à coopérer. Cette négociation va conduire à une révision, au moins partielle, de la stratégie de développement et de l'identité même de la commune ; celle-ci voit maintenant une opportunité dans cette découverte écologique et veut en faire le point de départ d'un développement touristique axé sur la nature et l'interprétation du milieu. Il y a bien ici ce que nous appelons une reconfiguration de la scène initiale : le conflit, par déplacements provoqués dans les sphères scientifique et politico-administrative, a créé les conditions d'une redéfinition de l'enjeu, d'un repositionnement des acteurs, bref d'une nouvelle manière de se représenter l'espace local, son avenir et son devenir. Le nouveau cadrage propose non seulement une nouvelle définition des enjeux mais crée aussi la possibilité de nouveaux échanges entre les partenaires locaux.

Il s'agit, dans ces exemples, de reconfigurations territoriales. De telles reconfigurations sont aussi possibles en termes de gestion (voir Aubin, 2004) ou en termes de référentiels professionnels et techniques : les multiples conflits ouverts ou implicites entre agriculteurs utilisateurs de pesticides et voisins peuvent conduire les arboriculteurs à chercher d'autres modèles techniques et professionnels pour leur production (Bergouignan et Cazals, 2004). Comme l'a montré Collet (Collet et Mormont, 2003), une telle redéfinition mobilise toutes les composantes de la chaîne agro-alimentaire, y compris le mode de communication entre producteurs et consommateurs.

\footnotetext{
3 Voir pour plus de détails Mougenot C (2003).
} 
Une fois cette reconfiguration effectuée, ce sont les préférences ${ }^{4}$ mêmes des agents qui sont modifiées puisque leur action dans le territoire est désormais recadrée et soumise à d'autres paramètres d'échange et d'évaluation. C'est un autre territoire qui se dessine ou se projette.

$\mathrm{Si}$ on raisonne brièvement en termes de politique publique il est clair qu'une telle dynamique est favorisée par des formes institutionnelles qui permettent une circulation des questions dans les différents espaces sociaux impliqués. C'est ici que prennent place les procédures de consultation, de concertation ou de délibération si souvent évoquées dans la littérature. L'adaptation des pratiques agricoles sous la pression des conflits de voisinage suppose que l'encadrement technique et économique de l'agriculture puisse être interpellé et soit capable de réagir en proposant de nouvelles techniques ou de nouvelles expérimentations. La rediscussion du projet d'implantation de la station d'épuration suppose que le système politico-administratif permette de réinterroger les critères techniques des bureaux d'étude ou les plans d'occupation des sols. Les recadrages ne sont possibles que si le questionnement des cadrages existants est autorisé. De même les processus que nous avons appelé de reconfiguration territoriale supposent qu'existent des espaces de discussion transversaux, à l'échelle du territoire.

\section{Territoires et agencements}

Dans cette dernière partie nous proposons de réinterroger la notion de territoire. Toutes les analyses qui portent sur les cadres de l'action collective privilégient une approche par la dimension cognitive. Mais les conflits environnementaux, s'ils questionnent les cadrages, c'est aussi en fonction d'enjeux substantiels, territoriaux. Car de quoi s'agit-il quand nous parlons de territoire? L'analyse sociologique classique, évoquée en début d'article, se centrait avant tout sur le territoire comme enjeu d'une compétition pour l'appropriation d'un espace, enjeu qui oppose des groupes sociaux dont les intérêts sociaux, économiques ou culturels les conduisent à utiliser de manières différentes cet espace. L'idée de territoire renvoyait donc à une possible identification entre des groupes sociaux et des espaces, le territoire étant la forme que prend un groupe social, une culture dans l'espace : ce sont des groupes et des cultures qui s'opposent donc dans l'espace enjeu des formes de territorialisation.

Nos analyses de conflits environnementaux, illustrées de quelques exemples stylisés, peuvent conduire à une autre grille de lecture. Dans une lecture classique de notre exemple de la station d'épuration, on chercherait évidemment à voir si des intérêts cachés ne sous-tendent pas les actions, par exemple si, comme cela a été souvent observé, ce ne sont pas plutôt des quartiers de classes moyennes qui protestent le plus efficacement contre des nuisances ou des risques, ce qui pourrait avoir pour conséquence de déplacer les pollutions et les dangers vers les espaces occupés par les groupes sociaux moins dotés de moyens de défense. L'argumentation de l'environnement ne serait là à nouveau qu'une rhétorique qui dissimulerait des intérêts particuliers. Dans une perspective un peu diffé-

\footnotetext{
${ }^{4}$ Par rapport à la plupart des analyses économiques, c'est donc plus à la construction sociale des préférences que le sociologue s'intéresse. Kirat et Levratto (2004) adoptent le même point de vue que nous : les conflits, dans la mesure où ils font inter-agir des interprétations différentes, modifient les préférences des agents. Une telle approche suppose d'abandonner l'individualisme méthodologique au profit d'une analyse qui privilégie l'institution comme unité d'analyse.
} 
rente on pourrait y voir un conflit entre un espace «aménités » privilégié par une population locale et un espace de services collectifs. Cette dernière perspective est souvent adoptée par les économistes quand ils distinguent des fonctions différentes de l'espace rural. Ces deux lectures ont en commun de relier une population (ou un groupe social localisé) à un territoire via un mode d'usage (ici un usage résidentiel), donc d'identifier espace et groupe social. Mais la dynamique de conflit environnemental que nous avons décrite ne se réduit pas à cela. En termes d'enjeux d'abord la station d'épuration est aussi un service qui bénéficie à cette population ainsi qu'à d'autres. On aurait donc plutôt affaire à un conflit de distribution des avantages et des nuisances. Mais surtout, cette dynamique est telle que l'action collective pousse ses protagonistes à chercher des alliances et des arguments généraux, à constituer des coalitions, à prendre en charge des intérêts multiples, des plus locaux à d'autres plus généraux : ce n'est donc pas une dynamique de privatisation des enjeux ni d'opposition entre groupes sociaux. La question que pose la localisation d'une station d'épuration ou celle que soulève le statut à définir d'une grotte abritant une espèce rare de chauve-souris pose une autre question qui est celle de savoir comment s'agencent territorialement des populations, des usages et des objets, qu'ils soient naturels ou techniques.

Ces questions appellent une autre lecture du territoire. Le territoire n'est plus dans ces exemples seulement un espace où se déploie un groupe social avec sa culture particulière, ses intérêts ou ses préférences. Nous faisons l'hypothèse qu'il faut trouver un autre vocabulaire de description et d'analyse de ces objets.

Ce qui est questionné dans les exemples étudiés c'est la présence dans un espace d'un « objet », naturel ou technique, qui vient le transformer et agir sur ceux qui y coexistent. En quoi cette présence est-elle problématique ? C'est qu'elle agit nécessairement sur eux. Car cette présence d'un «équipement » ou d'une espèce protégée, dans les deux cas appuyée sur de l'expertise scientifique ou technique, peut être définie comme un transformateur territorial. Peut-être faut-il alors décrire les territoires comme des espaces de transformation et pas seulement comme des espaces où se projettent des groupements humains.

La station d'épuration se laisse bien décrire comme un transformateur de flux : elle fait en effet circuler de l'eau, chargée de matières diverses et d'origines multiples, elle capte ces flux et les concentre pour les séparer, elle fait agir des populations de bactéries, elle fait interagir des composants chimiques ; enfin elle rejette de l'eau, des boues et alimente d'autres flux qui vont conduire ces matières vers d'autres espaces, d'autres recombinaisons. La station d'épuration est un objet qui connecte des flux et qui les transforme. Or chacun de ces flux dérive lui-même d'une série de transformations qui ont été opérées ailleurs dans des processus industriels, des modes de consommation, des systèmes de collecte des flux eux-mêmes.

Car la station doit être localisée, reliée à des réseaux, réseau d'égout et réseau hydrographique, si on veut qu'elle fasse ce qu'on attend d'elle (épurer), et aussi elle doit être connectée à un réseau de transformation et d'épandage des boues et toute cette circulation concrète compte.. Dans sa réalité technique, la station agence ces flux. Elle est donc agencement au sens d'action spécifique (combinaison / transformation des flux et des matières) et cela peut se lire à sa capacité de les transformer, les décomposer et les recomposer et donc de faire une différence entre ce qui entre et ce qui en sort. Sans cela elle n'est rien, elle est inefficace. L'agencement est donc multiplicité des flux, des forces et des matières 
mais c'est une multiplicité qui a pris une forme, une fonction, qui sont qualifiées par des opérations qui s'effectuent.

Enfin tout cela n'est possible qu'en faisant aussi circuler des flux financiers, des taxes, des crédits d'investissements, des redevances, des salaires, des royalties de brevets, bref toute une série de flux autres qui sont en bonne partie dépendants de normes et de connaissances situées ailleurs, opérantes dans d'autres « transformateurs de flux ». L'objet concret, la technique d'épuration, présuppose aussi des connaissances qui sont produites ailleurs et qui permettent de qualifier les flux, d'identifier les polluants, de décrire les recombinaisons que la station va effectuer. Cette notion d'agencement de flux et de processus de transformation en fait bien une réalité sociotechnique articulant des processus naturels et sociaux. Comment alors interpréter les conflits qu'elle suscite?

La station d'épuration réelle ne peut fonctionner qu'en étant dans un milieu, c'està-dire reliée à et reliant des flux qui lui sont extérieurs mais qui sont localisés. Chaque station réelle n'opère que dans un espace spécifique de circulation de ces flux. La station d'épuration ne sélectionne et ne qualifie que les flux qui sont dans le milieu. Ce faisant elle délimite un espace, la qualifie, le code... Elle crée en effet ce qu'on pourrait appeler le «bassin » d'épuration. Et ce bassin d'épuration est aussi un espace institutionnel de prélèvement des redevances sur les résidents, donc de circulation monétaire...

De plus, et c'est très important pour comprendre les conflits d'espace, cet agencement est ouvert : la station capte / rejette de/vers l'extérieur des flux, des matières, des gaz, et cette circulation conduit à d'autres agencements, par exemple celui de la ville, celui de la rivière, celui de l'agriculture qui accueille les boues comme engrais ${ }^{5}$. Ces flux sortants ouvrent sur d'autres territoires, sur des possibilités ou impossibilités de recombiner ces flux d'eau et de polluants avec d'autres transformations. Les boues d'épuration, il arrive qu'on ne sache trop quoi en faire, car les agriculteurs, échaudés par d'autres affaires d'importation de déchets dans la filière alimentaire, sont devenus méfiants ; dans le voisinage, les odeurs se répandent, et les voisins rouspètent, surtout les néo-ruraux dit-on; en outre certains polluants, ceux qui n'ont pas été qualifiés, transformés, recodés, se retrouvent quand même dans la rivière et inquiètent le monde de la pêche ou celui de la santé publique. Les externalités ne sont rien d'autre que les recombinaisons possibles de ces flux sortants avec d'autres. Mais les flux issus de la station changent de «nature » ou de caractéristiques au moment où ils sont repris par d'autres agencements. Bien sûr, si une zone humide permet de compléter l'épuration, on a un autre agencement avec des processus naturels : le flux des polluants résiduels est inscrit dans une nouvelle transformation, un autre «territoire». La topographie, le jeu des pentes et de l'hydrographie font d'ailleurs partie du fonctionnement de la station puisque celle-ci « bénéficie » de ces facteurs favorables à sa constitution même. Si par contre les odeurs perturbent le voisinage, affectent les valeurs immobilières, le «territoire » de la station entre en tension avec un territoire résidentiel. On pourrait interpréter de ce point de vue externalités positives et négatives dans ces termes. Les externalités positives ne sont elles pas ce qui s'échappe d'un agen-

\footnotetext{
${ }^{5}$ Par rapport au langage des économistes nous ferions alors l'hypothèse qu'on ne peut pas séparer la description économique d'une activité des multiples externalités qu'elle implique. Après tout il n'y a pas d'activité économique viable sans externalité. La station bénéficiera éventuellement des investissements qui ont été faits jadis dans un égouttage séparatif tout comme elle bénéficiera éventuellement de l'épuration tertiaire dans une zone humide préexistante qui accueille ses flux sortants...
} 
cement territorialisé pour être capté dans un autre agencement, dans un autre territoire ? Vu sous cet angle, il n'y pas d'activité (ou d'agencement) sans externalité. Toute activité capte des flux dont certains sont gratuitement offerts par d'autres activités et elle relance ses produits et sous-produits dans d'autres réseaux de circulation. Ce qui définit l'externalité c'est la recombinaison réussie - d'autant mieux réussie qu'elle n'est pas compensée par un flux monétaire - ou non dans d'autres activités.

Nous dirions alors que les conflits apparaissent là où les flux sortants rencontrent d'autres agencements sans pouvoir être recombinés positivement. Un autre territoire résiste. On ne peut comprendre ces conflits qu'en faisant le détour par les phénomènes de cadrage que nous avons évoqué. Les cadrages ce sont les processus de codage des flux : en effet, les matières, le bruit, les odeurs - qui sont bien des flux - sont susceptibles d'être perçus selon des codes différents. On s'en aperçoit bien en constatant la relativité des perceptions des nuisances selon les milieux (en fait les territoires) de réception, mais aussi les capacités d'internalisation, de recombinaison des matières dans d'autres « systèmes » de production ou de consommation. Les conflits émergent alors là ou des flux - par exemple un flux d'eau chargé d'un polluant qui a échappé à l'épuration - rencontrent des territoires (territorialisations) où ils sont codés différemment par d'autres agencements, où ils ne s'accordent pas aux cadrages des territoires d'accueil..

On peut faire le même genre d'analyse à propos de la grotte et des chauve-souris ; cet exemple a l'avantage de faire apparaître que l'agencement spécifique et local entre grotte et population animale, agencement de type écologique, peut lui-même être codé à différentes échelles, jusqu'à l'échelle internationale des listes d'espèces protégées définies par l'Union Européenne.

L'objet du conflit ne peut se comprendre, s'interpréter (et se gérer) qu'à condition de saisir que la station d'épuration n'est précisément pas un objet, ou plutôt qu'il n'est un objet (de conflit) que comme point de rencontre entre deux «agencements » ou encore de deux ensembles de pratiques qui agissent chacune sur ou avec la rivière. Et c'est l'action collective qui va conduire à l'enjeu du conflit, c'est-à-dire déterminer si c'est un conflit local d'usage (au cas où on peut adapter les lâchers d'eau aux exigences de la pêche) ou si c'est un conflit d'une autre dimension (au cas où il faudrait choisir entre le barrage ou la pêche). Il faut pour cela que l'action collective explore les deux ensembles de pratique, les connaissances qu'elles mobilisent, les normes auxquelles elles sont soumises, les acteurs qui peuvent en parler ou les modifier, bref que soient explorés leurs «cadres » et leurs « territoires».

Les conflits d'espace apparaissent alors autrement. Ce sont des points où des territorialisations, qui sont liées à des agencements, entrent en tension les unes avec les autres du fait des flux qu'elles entraînent d'un espace à l'autre. Les conflits, dans leur dynamique telle que nous l'avons exposée, manifestent ces tensions dans des frictions entre territorialisations. Or celles-ci sont à la fois codées (c'est le rôle des cadrages) et codantes (elles définissent les territoires): elles n'existent en effet comme telles que parce que, porteuses d'un mouvement, elles caractérisent le milieu où elles s'exercent. Les tensions se manifestent à propos d'objets - équipements, sites, paysages - et ces tensions font émerger les cadrages qui sont sous jacents à la définition des objets en cause. Et l'action collective va se déployer en suscitant des recadrages de ces mêmes objets. Quand les riverains interpellent les autorités sur le choix de localisation de la station d'épuration, ils se déplacent vers 
un autre territoire, celui de la commune (dont ils exploreront les potentiels pour une autre localisation), celui de la région (dont ils questionneront les choix techniques).

Les reconfigurations territoriales que nous avons évoquées comme possibles issues des conflits environnementaux ont aussi des effets sur les identités locales mais aussi sociales et professionnelles. Elles conduisent à redéfinir non seulement des relations de voisinage - des territoires - mais aussi des secteurs d'activité, à faire émerger de nouveaux projets ou à requalifier des pratiques en voie de disparition. Le domaine des relations entre agriculture et environnement (Lescureux F, Manouvrier E , 2004), la gestion de la biodiversité (Mougenot, 2003), les conflits autour des équipements et des infrastructures (Claeys-Mekdade C, 2003) sont quelques-uns des terrains privilégiés pour lire ces reconfigurations.

Dans cette dynamique, et c'est cela qu'il nous importe de souligner, ni les territoires, ni les groupes sociaux ne sont stabilisés : ils se redéfinissent réciproquement. Et c'est ce processus de redéfinition - jamais complètement terminée - qui nous paraît au cœur des conflits d'environnement dans l'espace rural.

\section{Conclusion}

Suivre la dynamique de conflits d'environnement, c'est suivre les actions collectives, suivre les acteurs dans les alliances qu'ils nouent, les reformulations de leurs arguments au long des déplacements qu'ils opèrent. Le concept de cadrage nous a servi a montrer que ces actions impliquent des transformations des connaissances, des normes et même des identités des acteurs. Ceux-ci ne sont donc pas donnés au départ de l'action, ils se construisent dans l'action, en même temps que se redéfinissent les questions, les problèmes et les solutions. Les cadrages sont alors moins des opérations stratégiques que des traductions au cours desquelles acteurs et objets de l'action s'entre-définissent. C'est que les cadrages sont bien des opérateurs de traduction. Les méthodes d'évaluation d'impact, les taux d'actualisation, tous les instruments « techniques » qui servent à définir et évaluer des projets sont à la fois le résultat de traduction et les opérateurs de nouvelles traductions.

L'impact de ces recadrages peut se lire dans les résultats de l'action collective, c'est-àdire ici principalement dans ce que nous avons appelé des reconfigurations territoriales.

Le vocabulaire analytique que nous avons ici proposé pour décrire ces reconfigurations emprunte à la philosophie de Deleuze et Guattari (1980). Le vocabulaire de celle-ci nous paraît ouvrir de nouvelles perspectives pour les analyses qui portent sur les territoires : en particulier ce vocabulaire met l'accent sur des processus plus que sur des entités essentialisées, sur le jeu des transformations et des traductions réciproques entre espaces, techniques et groupes humains. De plus, ce vocabulaire nous semble permettre d'articuler certains concepts économiques, comme l'analyse des externalités, à des concepts géographiques ou sociologiques.

Or la relation entre société et territoire ne nous semble plus pouvoir relever d'une analyse qui se limite à mettre en rapport des groupes sociaux et des espaces. En effet les questions d'environnement, parce qu'elles mettent à jour des articulations complexes entre des échelles différentes, entre des réalités hétérogènes, obligent à penser en termes de flux et de réseaux. Les réalités traditionnellement traitées par les sciences sociales, règles, identités et institutions, sont en fait inextricablement mêlées à des réalités matérielles, qu'elles soient biologiques, physiques ou techniques. Et qui plus est, elles sont également 
mobiles, en continuelle transformation par la circulation qu'elles assurent entre échelles et entre mondes différents. Les conflits sont une excellente entrée pour analyser ces changements. Il s'agit alors de suivre l'action telle qu'elle se déploie pour voir où et comment groupes sociaux et territoires se recomposent en faisant et défaisant des collectifs.

\section{Références}

Aminzade R., J. Goldstone, D. McAdam, E. Perry, W. Sewell, W. Tarro, Ch. Tilly (2001), Silence and Voice in the Study of Contentious Politics, Cambridge University Press.

Aubin D. (2004), Owners Escape Unharmed. Activation of Institutional Rules in Rivalries between Heterogeneous Water Users, communication to 10th IASCP Biennial Conference « The Commons in an Age of Global Transition: Challenges, Risks and Opportunities » Oaxaca (Mexico), 9-13 August 2004.

Belis-Bergouignan M.-C. et C. Cazals (2004), Conventions d'environnement de réseaux institués: prévention ou résolution des conflits d'usage ? Communication aux Journées « Conflits d'usage et de voisinage », Paris, 11 et 12 octobre 2004

Benford R., S. Hunt (2001), Cadrages en conflit. Mouvements sociaux et problèmes sociaux, in D. Trom, D. Cefaï, Les formes de l'action collective, Paris, Ed. de l'EHESS.

Bertrand N, S. Martin, N. Rousier (2004), Régulation des conflits d'usage de l'espace agricole péri-urbain. L'importance des documents d'urbanisme et de l'échelle de résolution des conflits, Communication aux Journées «Conflits d'usage et de voisinage », Paris, 11 et 12 octobre 2004 [ce numéro].

Boltanski L. (1975), Les usages sociaux de l'automobile : concurrence pour l'espace et accidents, Actes de la Recherche en Sciences Sociales, numéro $2: 25-49$.

Bourdieu P. (1979), La distinction. Critique sociale du jugement. Paris, Editions de Minuit.

Callon M. (1998), An essay on framing and overflowing : economic externalities revisited by sociology, in Callon M. The laws of the Markets, Oxford : Blackwell : 244-269.

Callon M. (1999), La sociologie peut-elle enrichir l'analyse économique des externalités ? Essai sur la notion de cadragedébordement, in D. Foray et J. Mairesse, Innovation et Performance, Paris, Ed. de l’EHESS, pp 399-431.

Cefaï, D. et D. Trom (2001, ed.), Les formes de l'action collective : mobilisation dans des arènes publiques, Paris, Ed. de l'EHESS, coll. Raisons Pratiques, n¹2, 2001.

Chamboredon J.-C. (1970) Proximité spatiale et distance sociale : les grands ensembles et leurs peuplements, Revue Française de Sociologie, 11, $1: 3-33$.

Chamboredon J.-C. (1985), La «naturalisation» de la campagne : une autre manière de cultiver les «simples» ?, in Cadoret A. (dir.), Protection de la nature: histoire et idéologie, Paris, L'Harmattan, pp.138-151.

Claeys-Mekdade C. (2003), Le lien politique à l'épreuve de l'environnement. Expériences camarguaises, Peter Lang, P.I.E., Bruxelles, 243p.

Collet E. et M. Mormont (2003), Managing pests, consumers and commitments: The case of apple and pear growers in Belgium's Lower Meuse region, Planning and Environment, volume 35 : 413-427.

Deleuze G. et F. Guattari (1980), Mille plateaux, Capitalisme et schizophrénie 2, coll. Critique, les Editions de Minuit.

Ejderyan O. (2004), Un paysage pour réduire les conflits ? Le cas de la participation du public dans les renaturations de cours d'eau en Suisse, Communication aux 4èmes journées de la proximité, Marseille, 17-18 juin 2004. 
Gamson W. (1985), Goffman's Legacy to Political Sociology, Theory and Society, 14, 5, p. 605-622.

Goffman E. (1974), Frame Analysis : An Essay on the Organization of Experience, New York, Harper. trad. fr. Isaac Joseph, Les cadres de l'expérience, Paris, Minuit, 1991.

Jeanneaux P. (2004), Economie de la décision publique et conflits d'usages dans les espaces ruraux et périurbains, Communication aux 4èmes journées de la proximité, Marseille, 17-18 juin 2004.

Kirat $\mathrm{T}$ et N. Levratto (2004), Les conflits d'aménagement, de l'utilité sociale à l'équité locale : le calcul économique et les dispositifs au défi de l'équité dans les nuisances des infrastructures, Communication aux Journées «Conflits d'usage et de voisinage », Paris, 11 et 12 octobre 2004.

Lescureux F. et E. Manouvrier (2004), Agriculture périurbaine et collectivité : des conflits d'usage et de voisinage à la coopération par l'intercommunalité de projet et la multifonctionnalité ? Le cas de la Communauté d'agglomération d'Hénin-Brevin (Nord-Pas-de-Calais), Communication aux Journées «Conflits d'usage et de voisinage », Paris, 11 et 12 octobre 2004.

Mallard A..et E. Remy (1999), Comment les associations renouvellent le débat sur la qualité de l'eau, Environnement et Société, $\mathrm{n}^{\circ}$ 22: 69-85.

Mormont M. (1983), The emergence of rural struggles, International Journal of Urban and Regional Research, $7,4: 559-575$.

Mormont M. (1990), Who is rural or how to be rural ? Towards a sociology of the Rural, in Rural Restructuring - Global processes and their responses (T. Marsden, P. Lowe and S. Whatmore Eds), London, Avebury, pp $21-44$.

Mormont M. (1980), Espace rural et domination : le cas des Ardennes belges, Sociologia Ruralis (Journal of the European Society for Rural Sociology), 10, 4 : 272-286.

Mougenot C. (2003), Prendre soin de la nature ordinaire, Paris : Inra et Maison des Sciences de l'Homme.

Nevers J.-Y. et S. Beccara (2004) : Les étangs du narbonnais : auto-régulation des usages, protection et mise en valeur d'un espace naturel, Communication aux Journées «Conflits d'usage et de voisinage », Paris, 11 et 12 octobre 2004.

Sencébé Y. (2001), Les lieux et les temps de l'appartenance. Mobilités et territoire : une analyse sociologique du pays Diois. Thèse de sociologie sous la direction de B. Ganne. Lyon : Université Lyon II, 3 tomes, 663 p. + Annexes.

Simmel G. (1908a) Soziologie. Untersuchungen über die Formen der Vergesellschaftung. Berlin, 1981 [trad. française de certains chapitres : Le conflit. Paris : Circé, 1992].

Simmel, G. (1908b), Soziologie. Leipzig, trad. française : Sociologie : études sur les formes de la socialisation. Paris : PUF, 1999.

Snow D. A., R. D. Benford (2000)., Framing Processes and Social Movements : An Overview and Assessment, Annual Review of Sociology, 2000, vol. 26.

Snow D., R. E. Burke, S. Worden, R. Benford (1986), Frame Alignment Processes, Micromobilization, and Movement Participation, AmericanSociological Review, 1986, 51, p. 464-481.

Trom D (1999), De la réfutation de l'effet Nimby considérée comme une pratique militante. Notes pour une approche pragmatique de l'activité revendicative, Revue française de science politique, 1999, vol. 49, $\mathrm{n}^{\circ} 1$, p. $31-50$. 